UNIVERSITATIS MARIAE CURIE-SKŁODOWSKA

\title{
Gauss curvature estimates for minimal graphs
}

\author{
Dedicated to the memory of Professor Jan G. Krzyż
}

\begin{abstract}
We estimate the Gauss curvature of nonparametric minimal surfaces over the two-slit plane $\mathbb{C} \backslash((-\infty,-1] \cup[1, \infty))$ at points above the interval $(-1,1)$.
\end{abstract}

1. Introduction. Statement of results. The relation between nonparametric minimal surfaces over simply connected domains and harmonic mappings is given by the Weierstrass Representation (see, e.g. [1]).

Theorem. A nonparametric surface $X$ over a simply connected domain $\Omega \neq \mathbb{C}$ is minimal if and only if there is a harmonic univalent and sense preserving mapping $f=h+\bar{g}$ of the unit disk $\mathbb{D}$ onto $\Omega$ such that its dilatation is the square of an analytic function. Moreover, $X$ can be represented as

$$
\left\{\left(\operatorname{Re} f(z), \operatorname{Im} f(z), 2 \operatorname{Im} \int b h^{\prime} d z\right): z \in \mathbb{D}\right\},
$$

where the dilatation $\omega(z)=g^{\prime}(z) / h^{\prime}(z)=b^{2}(z)$.

If the surface is a minimal graph and has a representation given in the Theorem, then the Gauss curvature at the point that lies over $w=f(z)$ is given by the formula (see [1] pp. 173-184)

$$
K(w)=-\frac{4\left|b^{\prime}(z)\right|^{2}}{\left|h^{\prime}(z)\right|^{2}\left(1+|b(z)|^{2}\right)^{4}} .
$$

2000 Mathematics Subject Classification. Primary 30C55, 53A10.

Key words and phrases. Harmonic mappings, minimal surfaces, Gauss curvature. 
If we apply the Schwarz-Pick lemma,

$$
\left|b^{\prime}(z)\right| \leq \frac{1-|b(z)|^{2}}{1-|z|^{2}}
$$

we get the estimates

$$
\begin{aligned}
|K(w)| & \leq \frac{4(1-|\omega(z)|)^{2}}{\left(1-|z|^{2}\right)^{2}(1+|\omega(z)|)^{4}\left|h^{\prime}(z)\right|^{2}} \\
& \leq \frac{4}{\left(\left|g^{\prime}(z)\right|+\left|h^{\prime}(z)\right|\right)^{2}\left(1-|z|^{2}\right)^{2}} .
\end{aligned}
$$

In particular,

$$
|K(f(0))| \leq \frac{4}{\left(\left|h^{\prime}(0)\right|+\left|g^{\prime}(0)\right|\right)^{2}} \leq \frac{4}{\left|h^{\prime}(0)\right|^{2}+\left|g^{\prime}(0)\right|^{2}} .
$$

If $X$ is a minimal graph above the unit disk $\mathbb{D}$ and $f$ is a harmonic map of $\mathbb{D}$ onto itself such that $f(0)=0$, then the Heinz lemma gives the estimate

$$
|K(0)| \leq \frac{16 \pi^{2}}{27}=5.848 \ldots
$$

Estimate (1.2) is not sharp. However, if one assumes that the minimal surface over $\mathbb{D}$ has a horizontal tangent plane at the point above the origin, then we have the sharp estimate

$$
|K(0)|<\frac{\pi^{2}}{2}=4.934 \ldots
$$

Proofs and further details may be found in [1].

Sharp bounds for Gauss curvature for minimal graphs over regions such as a half-plane, an infinite strip and the whole plane with a linear slit along negative real axis were found by Hengartner and Schober in [3]. S. H. Jun [4] obtained some estimates for the slit plane $\mathbb{C} \backslash[a, b]$.

We will use Hengartner-Schober [3] approach for the case of the plane with two linear slits along the real axis. We mention that harmonic mappings onto the two-slit plane were studied by Livingston [5] and Grigoryan and Szapiel [2].

Let $a<0<b$, and $\Omega(a, b)=\mathbb{C} \backslash((-\infty, a] \cup[b, \infty))$. A. Livingston considered the class $S_{H}(\mathbb{D}, \Omega(a, b))$ consisting of functions $f$ which are univalent, sense-preserving, harmonic mappings of $\mathbb{D}$ onto $\Omega(a, b)$, with normalization $f(0)=0, f_{z}(0)>0, f_{\bar{z}}(0)=0$. He proved that functions $f \in S_{H}(\mathbb{D}, \Omega(a, b))$ have the form

$$
f(z)=A\left[\operatorname{Re} \int_{0}^{z} \frac{\left(1-\zeta^{2}\right) P(\zeta)}{\left(1+c \zeta+\zeta^{2}\right)^{2}} d \zeta+i \operatorname{Im} \frac{z}{1+c z+z^{2}}\right]
$$

where

$$
A=b\left(\int_{0}^{1} \frac{\left(1-t^{2}\right) \operatorname{Re} P(t) d t}{\left(1+c t+t^{2}\right)^{2}}\right)^{-1}
$$


$P$ is analytic in $\mathbb{D}$, with $P(0)=1, \operatorname{Re} P(z)>0$ and $-2 \leq c \leq 2$. Moreover, Livingston showed that for given $a, b$ with $a+b \geq 0$ one can find numbers $c_{1}$ and $c_{2},-2<c_{1}<0<c_{2}<2$ such that $c$ in formula (1.3) is from the interval $\left[c_{1}, c_{2}\right]$. We will be interested in the symmetric case when $a=-b$. Then $c_{1}=-c_{2}$ (see [5]). Moreover, $c_{2}$ is the solution of the equation

$$
\sqrt{4-x^{2}}=2 \arctan \frac{x}{\sqrt{4-x^{2}}} .
$$

Indeed, by Lemma 1 in [5], $c_{2}$ is a unique zero of the function

$$
T(x)=\int_{0}^{1}\left(\frac{-\left(1+t^{2}\right)}{\left(1+x t+t^{2}\right)^{2}}+\frac{\left(1-t^{2}\right)}{\left(1-x t+t^{2}\right)^{2}}\right) d t, \quad-2<x<2,
$$

and a calculation shows that the zero of $T$ must satisfy (1.4). This means, in particular, that in the symmetric case $c$ in formula (1.3) lies in the interval $(-\sqrt{2}, \sqrt{2})$.

Our main results are the following theorems.

Theorem 1. If $X$ is a nonparametric surface over the two-slit plane $\Omega=$ $\mathbb{C} \backslash((-\infty,-1] \cup[1, \infty))$, then

$$
|K(0)| \leq \frac{\pi^{2}}{2} \frac{\left(1+\frac{8}{\pi^{2}}+\sqrt{1+\frac{32}{\pi^{2}}}\right)^{3}}{\left(1+\sqrt{1+\frac{32}{\pi^{2}}}\right)^{4}}=3.2642 \ldots
$$

Moreover, if $X$ has a horizontal tangent plane above the origin, then

$$
|K(0)| \leq \frac{\pi^{2}}{4} .
$$

Theorem 2. Under the assumptions of Theorem 1, the Gauss curvature at the point above $p \in(-1,1)$ satisfies

$$
|K(p)| \leq \frac{\pi^{2}}{2} \frac{\left(1+\frac{8}{\pi^{2}}+\sqrt{1+\frac{32}{\pi^{2}}}\right)^{3}}{\left(1+\sqrt{1+\frac{32}{\pi^{2}}}\right)^{4}} \frac{1}{(1-|p|)^{2}} .
$$

We also show that estimate (1.5) is sharp.

2. Proofs. In the proof of Theorem 1 we will use the following lemma due to Hengartner and Schober [3].

Lemma HS. If $b$ is an analytic function in $\mathbb{D}$ such that $|b(z)|<1$ for $z \in \mathbb{D}$, then

$$
\operatorname{Re}\left\{\frac{1+b^{2}(z)}{1-b^{2}(z)}\right\} \leq \frac{1}{2}\left(M\left(\frac{1+|z|}{1-|z|}\right)+\frac{1}{M}\left(\frac{1-|z|}{1+|z|}\right)\right)
$$

for all $z \in \mathbb{D}$, where $M=\max \left\{\frac{1-|b(0)|^{2}}{|1-b(0)|^{2}}, \frac{|1-b(0)|^{2}}{1-|b(0)|^{2}}\right\}$. This inequality is sharp for all real $z \in \mathbb{D}$ if and only if $b(z)= \pm \frac{z+\sigma}{1+\sigma z},-1<\sigma<1$. 
Proof of Theorem 1. If $f=h+\bar{g}$ maps $\mathbb{D}$ onto $\Omega$ and $f(0)=h(0)=0$, then

$$
\varphi(z)=h(z)-g(z)=\frac{\lambda z}{1+c z+z^{2}}
$$

with some $c,-\sqrt{2}<c<\sqrt{2}$. Moreover, we can assume that $\lambda>0$. Then $u(r)=\operatorname{Re} f(r)=f(r)$ is an increasing function that maps the interval $(-1,1)$ onto itself. Consequently, $1=\lim _{r \rightarrow 1^{-}} u(r)$ and $-1=\lim _{r \rightarrow-1^{+}} u(r)$. Since

$$
\operatorname{Re} f(z)=\operatorname{Re} \int_{0}^{z} \frac{\lambda\left(1-\zeta^{2}\right) P(\zeta)}{\left(1+c \zeta+\zeta^{2}\right)^{2}} d \zeta,
$$

where $P(z)=\frac{1+b^{2}(z)}{1-b^{2}(z)}, b^{2}(z)=g^{\prime}(z) / h^{\prime}(z)$, we get

$$
1=\lambda \int_{0}^{1} \frac{1-x^{2}}{\left(1+c x+x^{2}\right)^{2}} \operatorname{Re} P(x) d x
$$

and

$$
-1=-\lambda \int_{0}^{1} \frac{1-x^{2}}{\left(1-c x+x^{2}\right)^{2}} \operatorname{Re} P(-x) d x .
$$

Using the equality

$$
\varphi^{\prime}=h^{\prime}-g^{\prime}=\left(1-b^{2}\right) h^{\prime},
$$

we get from (1.1) that the Gauss curvature of the minimal graph over $\Omega$ above zero satisfies

$$
|K(0)| \leq \frac{4\left|1-b^{2}(0)\right|^{2}\left(1-|b(0)|^{2}\right)^{2}}{\left(1+|b(0)|^{2}\right)^{4}\left|\varphi^{\prime}(0)\right|^{2}} .
$$

It follows from (2.1) and (2.2) that

$$
\frac{1}{\lambda} \leq \int_{0}^{1} \frac{1-x^{2}}{\left(1+x^{2}\right)^{2}} \operatorname{Re} P(x) d x, \quad \text { if } c \geq 0
$$

and

$$
\frac{1}{\lambda} \leq \int_{0}^{1} \frac{1-x^{2}}{\left(1+x^{2}\right)^{2}} \operatorname{Re} P(-x) d x, \quad \text { if } c<0 .
$$

Now, using Lemma HS, we get

$$
\frac{1}{\lambda} \leq \frac{1}{2} \int_{0}^{1} \frac{1-x^{2}}{\left(1+x^{2}\right)^{2}}\left(M \frac{1+x}{1-x}+\frac{1}{M} \frac{1-x}{1+x}\right) d x,
$$

where $M$ is as in the Lemma HS.

Since $\varphi^{\prime}(0)=\lambda$, it follows from estimate $(2.3)$ that

$$
\begin{aligned}
|K(0)| \leq & \frac{\left|1-b^{2}(0)\right|^{2}\left(1-|b(0)|^{2}\right)^{2}}{\left(1+|b(0)|^{2}\right)^{4}} \\
& \times\left(\int_{0}^{1} \frac{1-x^{2}}{\left(1+x^{2}\right)^{2}}\left(M \frac{1+x}{1-x}+\frac{1}{M} \frac{1-x}{1+x}\right) d x\right)^{2} .
\end{aligned}
$$


Hence

$$
|K(0)| \leq\left(\frac{\left|1-b^{2}(0)\right|\left(1-|b(0)|^{2}\right)}{\left(1+|b(0)|^{2}\right)^{2}}\left(M \frac{\pi+2}{4}+\frac{1}{M} \frac{\pi-2}{4}\right)\right)^{2},
$$

where either $M=\frac{1-|b(0)|^{2}}{|1-b(0)|^{2}}$ or $M=\frac{|1-b(0)|^{2}}{1-|b(0)|^{2}}$. To complete the proof, we use the function $H$ defined in Lemma 5.2 by

$$
H(z ; \alpha, \beta)=\frac{\left|1-z^{2}\right|}{\left(1+|z|^{2}\right)^{2}}\left[\alpha \frac{\left(1-|z|^{2}\right)^{2}}{|1-z|^{2}}+\beta|1-z|^{2}\right] .
$$

It was shown in [3] that if $\alpha \neq \beta$ then $H$ assumes a maximum over $\mathbb{D}$ at $z_{0}=B\left[1-\sqrt{1-B^{-2}}\right]$, where $B=A\left[1+\sqrt{1+2 A^{-2}}\right]$ and $A=\frac{1}{2}\left[\frac{\alpha+\beta}{\alpha-\beta}\right]$. Moreover, the maximum value is

$$
H\left(z_{0} ; \alpha, \beta\right)=\frac{(\alpha+\beta)\left[1-B^{-2}\right]^{3 / 2}}{1-2 B^{-2}}
$$

if $\alpha \neq \beta$. Hence we get

or

$$
|K(0)| \leq \frac{1}{16} H^{2}(b(0), \pi+2, \pi-2)
$$

$$
|K(0)| \leq \frac{1}{16} H^{2}(b(0) ; \pi-2, \pi+2) .
$$

Since the maximum of both $H(\cdot ; \pi+2, \pi-2)$ and $H(\cdot ; \pi-2, \pi+2)$ is

$$
\frac{2 \pi\left(1-\frac{16}{\pi^{2}}\left(1+\sqrt{1+\frac{32}{\pi^{2}}}\right)^{-2}\right)^{3 / 2}}{1-\frac{32}{\pi^{2}}\left(1+\sqrt{1+\frac{32}{\pi^{2}}}\right)^{-2}},
$$

we get the desired estimate.

If $b(0)=0$, which means that the surface $X$ has a horizontal tangent plane at the point above the origin, we have

$$
|K(0)| \leq\left(2 \int_{0}^{1} \frac{1}{1+x^{2}} d x\right)^{2}=\frac{\pi^{2}}{4} .
$$

Remark. Under the assumption that the tangent plane is horizontal, inequality (1.5) is sharp. Actually, as in Livingston's paper one can consider the family $\mathcal{F}$ of harmonic mappings obtained by the shear construction of the functions

$$
\varphi(z)=\lambda \frac{z}{1+c z+z^{2}}
$$

for which equations (2.1) and (2.2) are satisfied. Then the family $\mathcal{F}$ contains all harmonic mappings $f$ of the disk $\mathbb{D}$ onto $\Omega$ such that $f(0)=0, f_{z}^{\prime}(0)>0$, $f_{\bar{z}}^{\prime}(0)=0$, and $\mathcal{F}$ is the closure of these mappings in the topology of the uniform convergence on compact subsets of $\mathbb{D}$. Taking $\varphi(z)=h(z)-g(z)=$ 
$\frac{\lambda z}{1+z^{2}}$ and the dilatation $\omega(z)=z^{2}$, we get from equation (2.1) (or (2.2)) that $\lambda=4 / \pi$ and we find $f \in \mathcal{F}$ of the form

$$
f(z)=\frac{4}{\pi}\left(\operatorname{Re}(\arctan (z))+i \operatorname{Im} \frac{z}{1+z^{2}}\right) .
$$

This function maps the unit disk onto the vertical strip $\{w:|\operatorname{Re} w|<1\}$. It lifts to a nonparametric minimal surface whose Gaussian curvature at the point above the origin is $\pi^{2} / 4$.

Since $f$ can be approximated uniformly on compact sets by harmonic mappings of the disk $\mathbb{D}$ onto $\Omega$ with dilatations $\omega_{n}=r_{n} z^{2}$, where $0<r_{n}<1$, $\lim _{n \rightarrow \infty} r_{n}=1$, we obtain a sequence of minimal surfaces over $\Omega$ whose Gaussian curvature above the origin converges to $\pi^{2} / 4$.

If we apply the shear construction to the same $\varphi$, but with the dilatation $\omega(z)=-z^{2}$, we get

$$
f_{1}(z)=\frac{8}{\pi}\left(\operatorname{Re}\left(\frac{z\left(1-z^{2}\right)}{2\left(1+z^{2}\right)^{2}}+\frac{1}{2} \arctan z\right)+i \operatorname{Im} \frac{z}{1+z^{2}}\right)
$$

which maps $\mathbb{D}$ onto $\Omega$. The function $f_{1}$ lifts to the nonparametric minimal surface whose Gaussian curvature at the point above the origin is $\pi^{2} / 16$.

Figures 1 and 2 depict the minimal surfaces above the harmonic shears $f$ and $f_{1}$, respectively.

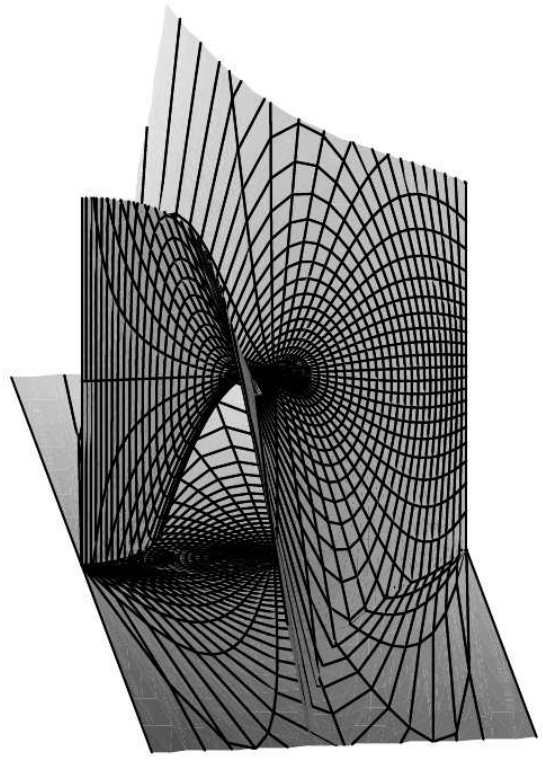

FiguRE 1. Minimal surface over a strip 


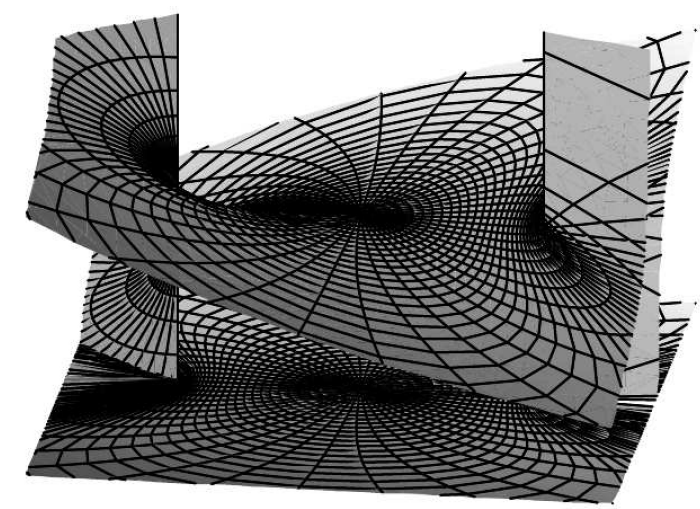

Figure 2. Minimal surface over the two-slit plane

Proof of Theorem 2. Assume that $f=h+\bar{g}$ is a harmonic map of the disk $\mathbb{D}$ onto $\Omega$ such that $f(0)=h(0)=p \in(0,1)$. If $\varphi=h-g$, then under the assumption that $\varphi^{\prime}(0)>0$,

$$
\varphi(z)=p+\frac{\lambda z}{1+c z+z^{2}}, \quad \lambda>0,
$$

where $\lambda$ and $c$ satisfy the equations:

$$
\lambda \int_{0}^{1} \frac{1-x^{2}}{\left(1+c x+x^{2}\right)^{2}} \operatorname{Re} P(x) d x+p=1
$$

and

$$
\lambda \int_{0}^{-1} \frac{1-x^{2}}{\left(1+c x+x^{2}\right)^{2}} \operatorname{Re} P(x) d x+p=-1,
$$

where, as above, $P=\frac{1+b^{2}}{1-b^{2}}, b^{2}=g^{\prime} / h^{\prime}$. Hence

$$
\frac{1}{\lambda}=\frac{1}{1-p} \int_{0}^{1} \frac{1-x^{2}}{\left(1+c x+x^{2}\right)^{2}} \operatorname{Re} P(x) d x \leq \frac{1}{1-p} \int_{0}^{1} \frac{1-x^{2}}{\left(1+x^{2}\right)^{2}} \operatorname{Re} P(x) d x
$$

in the case when $c \geq 0$, and

$\frac{1}{\lambda}=\frac{1}{1+p} \int_{0}^{1} \frac{1-x^{2}}{\left(1-c x+x^{2}\right)^{2}} \operatorname{Re} P(-x) d x<\frac{1}{1-p} \int_{0}^{1} \frac{1-x^{2}}{\left(1+x^{2}\right)^{2}} \operatorname{Re} P(-x) d x$

in the case when $c<0$. The rest of the proof runs as before. 


\section{REFERENCES}

[1] Duren, P., Harmonic Mappings in the Plane, Cambridge Univ. Press, Cambridge, 2004.

[2] Grigorian, A., Szapiel, W., Two-slit harmonic mappings, Ann. Univ. Mariae CurieSkłodowska Sect. A 49 (1995), 59-84.

[3] Hengartner, W., Schober, G., Curvature estimates for some minimal surfaces, Complex Analysis, Articles Dedicated to Albert Pfluger on the Occasion of his 80th Birthday, J. Hersch and A. Huber (eds.), Birhäuser Verlag, Basel, 1988, pp. 87-110.

[4] Jun, S. H., Curvature estimates for minimal surfaces, Proc. Amer. Math. Soc. 114 (1992), no. 2, 527-533.

[5] Livingston, A. E., Univalent harmonic mappings II, Ann. Polon. Math. 67 (1997), no. 2, 131-145.

Maria Nowak

Department of Mathematics

Maria Curie-Skłodowska University

pl. Marii Curie-Skłodowskiej 1

20-031 Lublin

Poland

e-mail: mt.nowak@poczta.umcs.lublin.pl

Received August 29, 2011

\author{
Magdalena Wołoszkiewicz \\ Department of Mathematics \\ Maria Curie-Skłodowska University \\ pl. Marii Curie-Skłodowskiej 1 \\ 20-031 Lublin \\ Poland \\ e-mail: woloszkiewicz@umcs.pl
}

